\title{
Clinicians' gaze behaviour in simulated paediatric emergencies
}

\author{
Ben McNaughten, ${ }^{1}$ Caroline Hart, ${ }^{1}$ Stephen Gallagher, ${ }^{2}$ Carol Junk, ${ }^{1}$ Patricia Coulter, ${ }^{1}$ \\ Andrew Thompson, ${ }^{1}$ Thomas Bourke ${ }^{1,3}$
}

${ }^{1}$ Department of Paediatrics, Royal Belfast Hospital for Sick Children, Belfast, UK

${ }^{2}$ Department of Behavioural Psychology, School of Psychology, Ulster University, Coleraine, UK

${ }^{3}$ Department of Medical

Education, Centre for Medical Education, Queen's University Belfast, Belfast, UK

\section{Correspondence to}

Dr Ben McNaughten, Royal Belfast Hospital for Sick Children, Belfast BT12 6BE, UK; bmcnaughten095@hotmail.com

Received 15 September 2017 Revised 18 January 2018 Accepted 9 February 2018 Published Online First 7 March 2018

\section{SLinked}

- http://dx.doi.org/10.1136/ archdischild-2018-315152

Check for updates

To cite: McNaughten $B$, Hart C, Gallagher S, et al. Arch Dis Child

2018:103:1146-1149.

\section{ABSTRACT}

Aim Differences in the gaze behaviour of experts and novices are described in aviation and surgery. This study sought to describe the gaze behaviour of clinicians from different training backgrounds during a simulated paediatric emergency.

Methods Clinicians from four clinical areas undertook a simulated emergency. Participants wore SMI (SensoMotoric Instruments) eye tracking glasses. We measured the fixation count and dwell time on predefined areas of interest and the time taken to key clinical interventions.

Results Paediatric intensive care unit (PICU) consultants performed best and focused longer on the chest and airway. Paediatric consultants and trainees spent longer looking at the defibrillator and algorithm (51 $180 \mathrm{~ms}$ and 50551 ms, respectively) than the PICU and paediatric emergency medicine consultants.

Conclusions This study is the first to describe differences in the gaze behaviour between experts and novices in a resuscitation. They mirror those described in aviation and surgery. Further research is needed to evaluate the potential use of eye tracking as an educational tool.

\section{INTRODUCTION}

During resuscitation, clinicians are required to absorb and process complex visual information in a time-critical manner. Eye-tracking technology allows for the measurement of an observer's point of gaze based on where their pupil is focused.

In aviation, differences in the gaze behaviour of experienced and novice pilots are clearly described. These differences exist across a variety of gazebased metrics including the number of times participants look at key areas (fixation count) and the duration of time spent looking at each of these areas (dwell time). Experts display shorter fixation times and more active visits to most instruments. They continue to look at key flight variables, including those not being manipulated, even during an emergency. ${ }^{1}$ A recent review identified differences in gaze behaviours between expert and novice surgeons and cited the potential use of eye-tracking technology as an educational tool. ${ }^{2}$

Our literature review identified three studies assessing the use of eye-tracking technology in the resuscitation setting. One observational study explores the potential of mobile eye tracking in nursing and paramedic students as a debriefing tool. $^{3}$ The second describes a technical feasibility study considering the use of gaze tracking in

\section{What is already known?}

- Research in the aviation industry and surgery has demonstrated differences in the gaze behaviour between experts and novices.

- It can be used as a part of a targeted intervention to improve performance.

\section{What this study adds?}

- The use of eye tracking technology during simulated resuscitations is feasible.

- Our study demonstrates a difference in the gaze behaviour of experts and novices in a simulated emergency.

- This technology has real potential as an educational tool.

medical simulation. ${ }^{4}$ The third study describes the gaze behaviour of six clinicians during neonatal resuscitation. $^{5}$

Novice pilots are taught the eye gaze pattern of experts early in their training. We believe similar strategies could be used in resuscitation training. The aim of this study is to describe the eye gaze pattern of experts and novices to inform further study into using these techniques as an educational strategy.

\section{METHODS}

We designed an observational cohort study to explore gaze behaviour and performance of clinicians in a simulated emergency.

\section{Sample}

A convenience sample of paediatric trainees, and paediatric, paediatric intensive care unit (PICU) and paediatric emergency medicine (PEM) consultants participated.

\section{Procedures}

The study took place at the Royal Belfast Hospital for Sick Children in October 2016. The simulation suite contained a Laerdal SimJunior and was designed to mimic clinical areas. Recognised algorithms for common emergencies were available.

Participants received an orientation to the simulation suite. The eye-tracking equipment was fitted and calibrated. Each participant undertook the same, standardised 6 min scenario based on a 
4-year-old boy in ventricular fibrillation cardiac arrest following a tricyclic antidepressant overdose. Participants were blinded to the scenario.

\section{Data collection}

Gaze behaviour

Participant's eye movements were recorded using SMI (SensoMotoric Instruments) eye tracking glasses (figure 1). These allowed the participant's pupil fixation to be overlaid on a simultaneous point-of-view recording. During data analysis, the eye gaze fixations were represented by coloured dots on the video (figure 1). The equipment was run using 'iView ETG' software. Data were analysed using 'BeGaze V.3.4'.

We identified four key areas of interest before the study: (1) airway, (2) cardiac arrest algorithm, (3) chest and (4) defibrillator.

We analysed the fixation count and dwell time on each of these areas of interest.

\section{Time to key clinical interventions}

The time taken to bag and mask ventilation, chest compressions and first shock were recorded.

\section{RESULTS}

We recruited 28 participants. We were unable to calibrate the equipment for one participant leaving 27 participants: eight consultant paediatricians, seven PICU consultants, six PEM consultants and six paediatric trainees.

\section{Gaze behaviour}

The mean fixation count and dwell time of the participants in each group are shown in figure 2. Participants from all groups looked most often and for longer at the patient (chest and airway) than any other areas of interest. However, the mean fixation count (396) and dwell time (96286 ms) on the patient's chest and airway was noticeably longer for PICU consultants. The gaze behaviour of the paediatric consultants and trainees was similar. The combined average total dwell time on the defibrillator and algorithm for the paediatric consultants and paediatric trainees were $51180 \mathrm{~ms}$ and $50551 \mathrm{~ms}$, respectively. This compares to $28095 \mathrm{~ms}$ for the consultants in PEM and $19804 \mathrm{~ms}$ for the PICU consultants.
The Binning charts displayed in figure 3 provide a visual representation of the gaze patterns of a paediatric and PICU consultant. Each line represents a period of two seconds. The colour of the line represents the area of interest which the participant was looking at during that period. The height of the line represents the duration of the gaze with a shorter line representing a shorter glance.

Both participants initially focus on the airway and chest. The consultant paediatrician then spends a large proportion of time looking at the defibrillator and algorithm. The PICU consultant continues to look at all areas of interest for shorter periods of time. The chart is noticeably less densely populated reflecting greater proportions of time when the participant is not looking at one of the key areas of interest. Review of the POV recordings shows that much of this time is spent communicating with team members and surveying the wider resuscitation area.

\section{Time to key interventions}

Table 1 shows the median time taken to perform key clinical interventions.

\section{DISCUSSION}

Our study is the first to describe the gaze behaviour of expert and novice clinicians in a simulated emergency. PICU consultants performed best and could be considered experts. Their gaze behaviour differed to that of other groups. Most noticeable was the high mean fixation count and dwell time on the patient's chest and airway. They also paid more attention to all areas of interest for shorter periods of time.

This gaze behaviour is similar to that observed in experienced pilots who make shorter fixations, more active visits to important instruments and more checks on flight variables not being manipulated. ${ }^{1}$ PICU consultants are more likely to encounter time critical emergencies in their practice. Pilots on short-haul flights perform up to five times more landings than those on long-haul flights and demonstrate superior gaze behaviour. ${ }^{1}$

In contrast, consultant paediatricians performed worst in time to compressions and first shock. This may be due to less exposure to shockable cardiac arrests than their PICU and PEM colleagues. They demonstrated similar gaze patterns to true novices (trainees). They looked more frequently and for longer

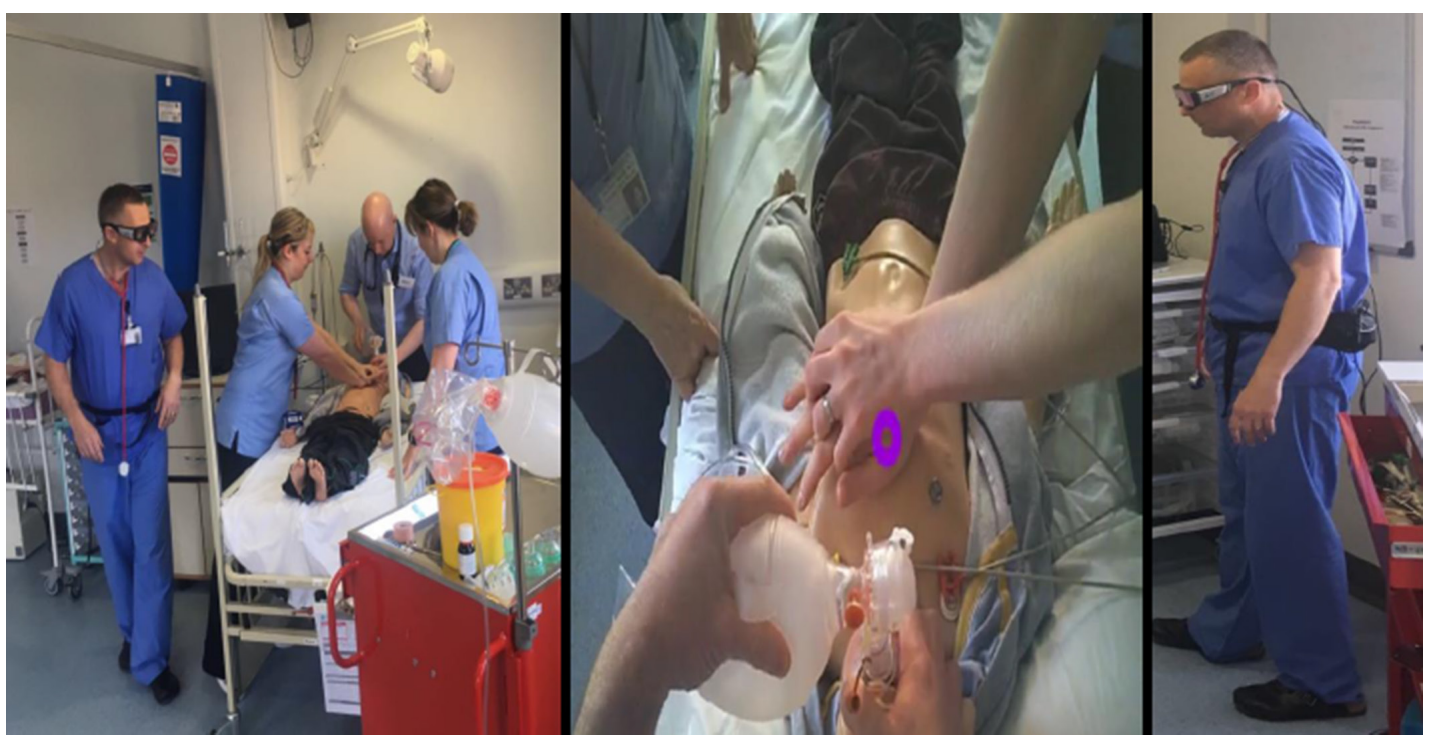

Figure 1 Candidate participating in simulated scenario and view from the SMI eye tracking glasses used during the study. 

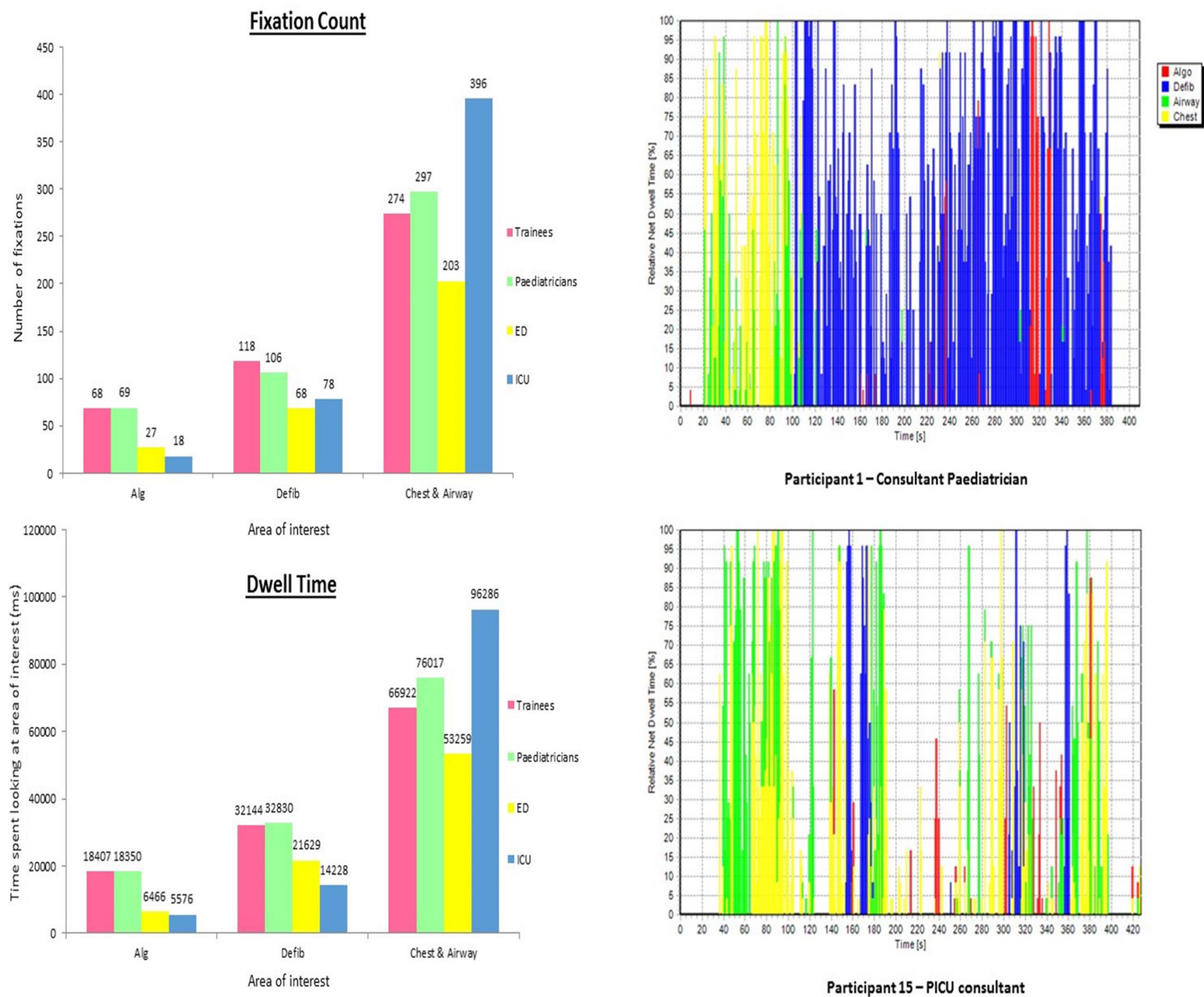

Figure 2 Graphs displaying the mean fixation count and dwell time of particpants from each group on the pre-defined key areas of interest.

at the algorithm and defibrillator than other clinicians. This type of 'perceptual narrowing' in situations of high cognitive load has been described in novice drivers and increases the risk of missing key visual cues.

We found that eye tracking in simulated resuscitations is technically feasible. Recordings were of good quality and participants found the glasses comfortable.

The small size and convenience nature of the study is the main limitation. This was a simulated emergency, and it is impossible to know without further study if an individual's performance and gaze behaviour accurately reflects their normal clinical practice. We did not assess visual scan patterns which may have provided additional useful information.

\section{CONCLUSION}

Our study demonstrates a difference in gaze behaviour between experts and novices. Evidence from aviation and surgery supports the use of gaze tracking as a targeted intervention to improve trainee's performance. ${ }^{12}$ We believe that these methods have real potential as a tool to modify and improve clinicians

Figure 3 Binning charts representing the gaze behaviour of one consultant paediatrician and one paediatric intensive care unit (PICU) consultant.

Table 1 Median time and range in seconds

\begin{tabular}{lrcr}
\hline & $\begin{array}{l}\text { Bag and mask } \\
\text { ventilation }\end{array}$ & $\begin{array}{l}\text { Chest } \\
\text { compressions }\end{array}$ & \multicolumn{1}{c}{ First shock } \\
\hline Trainees & $29.5(23-57)$ & $47(13-78)$ & $129(120-154)$ \\
$\begin{array}{l}\text { Paediatricians } \\
\begin{array}{l}\text { Paediatric } \\
\text { emergency medicine }\end{array}\end{array}$ & $34(31-49)$ & $60.5(40-110)$ & $142(94-210)$ \\
$\begin{array}{l}\text { Paediatric intensive } \\
\text { care unit }\end{array}$ & $27(25-48)$ & $43.5(25-63)$ & $123.5(85-155)$ \\
& & $32(26-53)$ & $105(90-144)$ \\
\end{tabular}

performance during time critical emergencies. Further study is required to evaluate if this approach adds value to traditional training methods.

Acknowledgements The authors would like to thank Ross Christie and Greg Barton for their assistance in data analysis. In addition we would like to thank Dr Sam Lamont for granting his consent for photographs of his scenario to be used in the publication. We are also very grateful to the Medical Staffing Committee in RBHSC for funding Dr McNaughten's fellowship 
Contributors BM wrote the first draft. All other authors reviewed the manuscript and agreed on the final version for submission.

Funding This research received no specific grant from any funding agency in the public, commercial or not-for-profit sectors.

Competing interests None declared.

Patient consent Not required.

Ethics approval Belfast Health and Social Care Trust Governance Department.

Provenance and peer review Not commissioned; externally peer reviewed.

(c) Article author(s) (or their employer(s) unless otherwise stated in the text of the article) 2018. All rights reserved. No commercial use is permitted unless otherwise expressly granted.

\section{REFERENCES}

1 Haslbeck A, Zhang B. I spy with my little eye: Analysis of airline pilots' gaze patterns in a manual instrument flight scenario. Appl Ergon 2017;63:62-71.

2 Hermens F, Flin R, Ahmed I. Eye movements in surgery: a literature review. Journal of Eye Movement Research 2013;6.

3 Browning M, Cooper S, Cant R, et al. The use and limits of eye-tracking in high-fidelity clinical scenarios: A pilot study. Int Emerg Nurs 2016;25:43-7.

4 Szulewski A, Howes D. Combining first-person video and gaze-tracking in medical simulation: a technical feasibility study. Scientific World J 2014;2014:1-4.

5 Law BHY, Cheung PY, Wagner M, et al. Analysis of neonatal resuscitation using eye tracking: a pilot study. Arch Dis Child Fetal Neonatal Ed 2018;103:F82-4. 